\title{
A COMPARISON OF ISDN AND LAN TECHNOLOGIES
}

\section{FOR SHIPBOARD USE}

\author{
Randall J. Atkinson \\ Information Technology Division \\ Naval Research Laboratory \\ Washington, DC 20375
}

\begin{abstract}
This paper discusses the use of ISDN, B-ISDN, and FDDI for use as link protocols for use aboard ship. Connectionless services and connection-oriented services are compared and their appropriateness for use in a tactical environment is analysed. Conclusions are drawn on which technology is most appropriate for shipboard and for use between land-based command centers.
\end{abstract}

\section{Introduction}

Recently there has been significant US Navy interest in using optical networking technology aboard ship to increase operational capabilities by integrating shipboard systems and providing greatly enhanced communications capabilities. The Navy plans to deploy SAFENET, an FDDI-based LAN, aboard ships within the next few years. [1,2] The Communications Support System (CSS) project is looking at Navy communications needs to devise a more effective communications architecture. There are several competing concepts for CSS, one of which uses ISDN technology both onshore and aboard ship. Other more researchoriented efforts such as the NRL High-Speed Optical Network project are looking at next generation Broadband ISDN (B-ISDN) technologies that might be used near the end of this decade. This paper compares ISDN, Broadband ISDN (B-ISDN), and FDDI technologies for use in shipboard networks.

\section{Topology \& Cabling}

ISDN primarily uses copper cables to connect each terminal device to a nearby switch and copper cables between switches. B-ISDN, including the Synchronous Optical Network (SONET) and Asynchronous Transfer Mode (ATM) standards, instead uses fiber-optic cabling. Both ISDN and B-ISDN use the "star" topology with a central switch and nodes connected to that switch. If a switch fails, all nodes connected to it become disconnected from the network. The only way to prevent such catastrophic failures is to have a pair of switches so that a hot backup can take over if the primary fails. This redundancy is expensive.

By contrast, FDDI uses multiple dual-counter-rotating fiber-optic rings to connect nodes. There is less cable required be- cause of the difference in topology. The ring will quickly and automatically reroute around any single cable cut. If a connected node fails, this is automatically detected by the wiring center for that node and the rest of the ring automatically routes around that node without any need for human intervention. FDDI rings have high survivability and no single point of failure. FDDI is possibly better at joining other existing LANs together because they all use the common IEEE 802.2 Logical Link Control protocol and share a common address space. In such environments, the sharing of the bandwidth can mean that it is used more efficiently than if ATM were used.

\section{Data Rates}

The existing base ISDN data rate is $64 \mathrm{Kbps}$ and the fastest common ISDN circuit is the T3 at 45 Mbps. ATM networks currently have data rates of around $150 \mathrm{Mbps}$ but can scale up past $1 \mathrm{Gbps}$. The data rates in ISDN and B-ISDN are usable point-topoint between two nodes in the network, unlike FDDI where that bandwidth is shared by all of the nodes on the ring. This makes ATM more attractive than FDDI for handling high data rate traffic such as sensor data, audio, or video.

FDDI currently has a $100 \mathrm{Mbps}$ data rate and the FDDI Follow-On Lan (FFOL) will move this past $1 \mathrm{Gbps}$. While ISDN has a much slower data rate, ATM has $150 \mathrm{Mbps}$ data rates now and will have Gbps data rates within a few years. The FDDI II effort that is underway will add full support for isochronous and pleisiosynchronous traffic, including bandwidth allocation mechanisms for critical applications. FFOL appears unlikely to ever have the same aggregate bandwidth as an ATM network and this would be a problem for applications that require dedicated gigabit bandwidth but can be an advantage when used as a backbone to join existing LANs together. ATM appears to be maturing more quickly than FDDI-II or FFOL. However, SONET is not maturing as quickly as ATM or FDDI for use in data communications.

\section{Fault Tolerance}

ISDN and other connection-oriented services do not handle faults as well as connectionless services do. Switch conges-

\subsection{1}


tion is the most common source of data loss in ISDN and B-ISDN networks. Because of the centralised topology, each switch is a critical single point of failure. Moreover, switches are so complicated that it is difficult or impossible to formal assure the correctness of either design or implementation. When a fault occurs, the connection is broken and the communicating parties must open a new connection. Also, connection setup and restoration normally takes a long time, compared with transmission rates. So many ISDN and ATM testbeds are experimenting with permanent virtual circuits, which remove some of the call setup overhead costs of switched virtual circuits. Ways to eliminate or substantially reduce the problem with circuit setup and restoration need to be discovered and employed for connection-oriented services to meet tactical requirements.

By contrast if connectionless services are used below a connection-oriented transport service, the connection can continue to work properly despite faults at lower layers. If the transport layer connection is lost due to a fault, it can be reopened much more quickly since only one negotiation is required. Some more recent transport protocols, such as XTP permit data to be sent in the same packet as the request to open or reopen a transport connection, which is a substantial improvement over some other transport protocols.[3] The FDDI topology and protocols do not suffer from the problems of connection-oriented link layers and FDDI has mechanisms to automatically wrap the ring to keep any single fiber cut from severing connectivity. For ATM networks, use of a mesh architecture of switches connected with permanent virtual circuits to reduce vulnerability appears promising. Experience with failures related to the ISDN control protocol shows that the proper design of the control mechanisms and protocols for ATM is critical. CCITT has not yet standardised on such mechanisms. It isn't clear that ATM will be really useful until it has fully defined signalling and control protocols.

\section{Control Protocols}

The widespread problems with commercial telephone service in the Mid-Atlantic area of the US during July 1991 were due to errors in only 2 lines of software implementing the Signalling System 7 (SS7) control protocol in a switch. The faulty implementation within the one system caused other systems to fail. There are concerns within the protocol verification community that SS7 might have fundamental protocol design errors. Even officials of Bell Atlantic have expressed concern about the correctness of the SS7 protocol specification. Bellcore's investigation reportedly determined that "the design of the congestion-control mechanism within each [switch] contributed to the failure by allowing the congestion to back up through multiple [switches]." [4].

Most existing physical and link layer protocols are vulnerable to attack by anyone with access to the network. However, ISDN is unusually vulnerable because of its control protocol. The widely used Signalling System 7 (SS7) control protocol does not provide suitable security properties for its control messages. [5] ISDN relies on physical security of the cabling and switches to prevent someone from gaining access to the control channel.
Aboard ship it is difficult to entirely protect the cabling. Onshore networks are even more difficult and expensive to physically secure. The use of link encryption over the RF circuits is of limited value because almost any node in the ISDN network could potentially be used to break into or shut down the network or selected portions of the network. If any node were penetrated, all of the other nodes on the ISDN network might be vulnerable.

It is difficult to provide general authentication or confidentiality properties below the network layer using existing protocols and standards. The only readily-available network layer protocol having security properties is SP3. SP3 provides connection-less network service, meaning that the connection-oriented link layer service below it would incur additional costs without providing any real benefits. The use of link cryptography throughout the network appears to be necessary but would require custom ISDN switch hardware. ISDN vendors appear to have little interest in securing the critical SS7 control protocol.

The Station Management (SMT) protocol of FDDI also lacks any authentication or confidentiality. However, this is much less of a problem because SMT primarily provides network status and monitoring information rather than control over circuits, virtual circuits, or remote nodes (because FDDI doesn't provide virtual circuit services). Moreover, the SMT implementation at each FDDI node can be used to help detect and trace denial-of-service attacks. Although SMT is not ideal, it has many fewer security problems than SS7.

\section{Performance}

A connection-oriented service provides more features to the service user than a connectionless service would provide at the same ISO OSI layer. However, this does not mean that it is better to use connection-oriented services at every layer. Rather, it means that a connection-oriented service should be available at the transport or session layers. Connection-oriented protocols are much more complicated, making them more difficult to design and implement correctly. For example, early specifications of the CCITT X.21 protocol had fundamental protocol design errors that went undetected for many years. Eventually someone tested protocol verification software on the CCITT X.21 protocol and discovered the protocol design error. [6] The call setup time and call tear down time required for connection-oriented services hampers performance; connection-less services do not have these costs. Connection oriented services at the lower layers will take longer to recover from faults than connectionless services would.

\section{Routing}

At the network layer, dynamic routing is a significant advantage over static or operator-configured routing. Dynamic routing is the automatic and dynamic rerouting of communications traffic over diverse links as necessary to avoid congestion and other faults in order to deliver the traffic reliably. During Operation Desert Storm, Iraq successfully used dynamic routing of communications traffic to significantly minimise the effects of US bomb- 
ing attacks on their ability to communicate with and control their forces.

Connection-oriented networks such as ISDN and most forms of B-ISDN usually route only once, at call setup time, and do not change that route. More recently, ISDN providers have permitted human operators to reroute circuits. However, circuit rerouting is not automatic in ISDN or B-ISDN and therefore requires humans to be present at the network control point. If a fault occurs, the traffic will not automatically be rerouted around it but instead will be dropped. Human intervention is also necessary to fully balance load among various links in the network. Connectionless networks do all this automatically without human intervention and with minimal loss of data.

Researchers are currently working on how best to handle addressing and routing in B-ISDN networks, with most of the work concentrating on ATM addressing and routing. The current standards are very connection-oriented. It is unclear whether useful connectionless specifications will arise in the near term. The interest of the Internet Engineering Task Force (IETF) and the ATM Forum in using ATM to support connectionless networking means that ATM is the most promising ISDN or B-ISDN technology for use in tactical environments. By contrast, FDDI was designed to handle connectionless networking and doesn't have these problems.

\section{Summary}

The use of connection-oriented protocols below the transport layer is inadvisable in shipboard environments if alternative connection-less lower layer architectures are viable. Use of connection-oriented lower layer architectures tends to increase equipment cost and vulnerability to faults. Use of permanent virtual circuits may reduce this problem for ATM networks. FDDI networks are ideal for connecting existing LANs together and have properties making them especially well suited for shipboard use. The use of ISDN and B-ISDN services ashore between command centers and other such installations is desirable for economic reasons and reasonable because the operating environment is less hostile. Many telephone companies are either refusing to lease fiber directly or are making it prohibitively expensive. Instead the telephone companies only wish to sell value-added services that use fiber optic cabling, so being able to use those value-added services to support data networking is important.

\section{Bibliography}

[1] NCCOSC, Survivable Adaptable Fiber Optic Embedded Network (SAFENET), MCCR-0035, Draft 2, (Draft MILSTD), NCCOSC, San Diego, CA, 10 January 1992.

[2] NCCOSC, SAFENET Network Development Guidance, MCCR-0036, Draft 2, (Draft MIL-HDBK), NCCOSC, San Diego, CA, 10 January 1992.

[3] Chesson, Greg, XTP Protocol Specification, Revision 3.6, January 1992, Protocol Engines Incorporated, Santa Monica, CA.

[4] Burgess, Angela, In the News, IEEE Software, May 1992, p. 105

[5] CCITT, CCITT Blue Book, Volume VI, Fascile VI.7, 1988.

[6] Holzmann, Gerard, Design and Validation of Computer Protocols, Prentice-Hall, Englewood Cliffs,NJ, 1991. 